\title{
Critical analysis of the 'generalized coherent wave approximation'
}

\author{
T. C. MCGILL† and J. KLIMA \\ H. H. Wills Physics Laboratory, Bristol University, Bristol BS8 1TL. \\ MS. received 11 th May 1970
}

\begin{abstract}
The formalism developed by Fletcher to take account of the presence of short range order in the calculation of the electronic energy spectrum of amorphous covalent semiconductors is examined critically and found to have fundamental difficulties.
\end{abstract}

\section{Introduction}

Short range order is widely held to be responsible for the presence of an energy gap in elemental amorphous covalent semiconductors. Consequently, theoretical interest has developed in showing the connection between these two facts. One promising attempt, which has received rather widespread attention, is reported in a series of papers by Fletcher $(1967 \mathrm{a}, \mathrm{b}, \mathrm{c})$. Numerical calculations based on this formalism which have been made in this laboratory and others, have been largely unsuccessful and have led the authors to undertake a critical analysis of this formulation of the problem. As a result of this study, the authors have noted certain fundamental difficulties both with the formalism and the results. These errors are of sufficient magnitude to call into serious question the validity of this approach.

\section{Review}

To put the remarks contained in this paper in proper perspective, the main points of Fletcher's quantative work (Fletcher 1967 b) are reviewed. In this paper a formalism for a two dimensional model with strong short range angular correlations but total absence of long range order is developed. Each centre is assumed to be surrounded by $Z$ nearest neighbours. The formalism is based upon a generalization of the 'coherent wave' approximation introduced by Phariseau and Ziman (1963) to facilitate the calculation of the density of states of a liquid metal. The 'coherent wave' approximation assumes that the 'average' wave function about one site is related to that about another site by a transformation analogous to a Bloch transformation.

The generalization consists of the introduction of an additional transformation. This transformation adapts the wave function about the various sites to the differences in the angular orientation of the spatial location of its nearest neighbours. That is

$$
\psi_{j}^{\alpha_{j}}(\boldsymbol{\rho})=\exp \left\{\boldsymbol{i} \boldsymbol{k} \cdot\left(\boldsymbol{x}_{j}-\boldsymbol{x}_{i}\right)\right\} R\left(\alpha_{j}-\alpha_{i}\right) \psi_{i}^{\alpha_{i}}(\boldsymbol{\rho})
$$

(equation (13), Fletcher $1967 \mathrm{~b}$ ), where $\psi_{j}^{\alpha_{j}}(\rho)$ is the wave function about site $j$ with angular orientation of nearest neighbours $\alpha_{j}, x_{j}$ is the location of site $j . R(\alpha)$ is the additional transformation which is called the generalized rotation operator. In line with an intuitive approach to the problem, $R(\alpha)$ is assumed to satisfy the relations

and

$$
R(\alpha) R(\beta)=R(\beta) R(\alpha)=R(\alpha+\beta)
$$

$$
R(2 \pi S / Z)=1 \quad S=0,1,2 \ldots
$$

+ NATO Postdoctoral Fellow. 
(equations (14) and (15) Fletcher 1967 b). Equation (2) is the commutativity and additivity relationship satisfied by standard rotations in two dimensions, and equation (3) reflects the fact that a rotation of $2 \pi / Z$ brings the nearest neighbour structure to an orientation which is indistinguishable from its original orientation.

Because of these conditions, $R(\alpha)$ is taken to have the form

$$
R_{m m^{\prime}}(\alpha)=\delta_{m m^{\prime}} \sum_{n} a_{n, m} \exp (\mathrm{i} n Z m \alpha)
$$

(equation (17), Fletcher $1967 \mathrm{~b} \dagger$ ), where $m$ refers to the basis $\{\exp (i m \phi) / \sqrt{ } 2 \pi\}$ and $a_{n, m}$ are coefficients which are believed to be undetermined by the assumed properties of $R(\alpha)$.

The development of the formalism proceeds in the manner analogous to the standard KKR method (Kohn and Rostoker 1954), adapted for two dimensions and averaged over the disorder using techniques similar to those of Phariseau and Ziman (1963). The wave function about a site is expanded in the basis $\{\exp (\operatorname{im} \phi) / \sqrt{ } 2 \pi\}$

$$
\psi(\rho)=\sum_{m} b_{m} R_{m}(\rho) \exp (\operatorname{im} \phi)
$$

where $R_{m}(\rho)$ is the solution of the radial Schrödinger equation for the single site potential. However, the variational step in this calculation differs in two fundamental ways from the analogous step in the standard KKR formalism. First, in this formalism the product (labeled $\beta_{n m}$ ) of the undetermined coefficients in the expansion for the rotation operator, $a_{n, m}$, with the coefficients in the expansion of the wave function, $b_{m}$, are varied. This should be contrasted with the standard approach in which only the coefficients in the expansion for the wave function are varied.

Further, the set of $\beta_{n, m}$ is usually larger than the set of $b_{m}$. For, if $M$ is the number of $m$ values taken in the expansion of the wave function, and $N$ is the number of $n$ values taken in the expansion of the rotation operator, then the set of $\beta_{n, m}$ is $N$ times as large as the set of $b_{m}$.

Second, a condition for the attainment of an extremum of the variational functional (equation (27), Fletcher 1967 b) which results in only $M$ linearly independent equations is used. Since there are $N \times M$ unknowns in these equations, it is necessary to generate $N-1$ additional equations from each equation in this set. This is accomplished by taking different values for the orientation of the nearest neighbours about the central site. In contrast, in the standard KKR formalism all of the required number of linearly independent equations are generated by the condition for an extremum of the variational functional.

The results of the variational step when $N=2$ and $Z$ is odd, is a set of homogeneous equations for the $\beta_{n, m}$. Using a notation in which $\beta_{0}$ and $\beta_{1}$ are column vectors with elements $\beta_{0, m}$ and $\beta_{1, m}$, respectively, the set of equations may be written in the form

$$
\left(\begin{array}{cc}
\mathbf{F}^{0} & \mathbf{F}^{1} \\
\mathbf{F}^{0} & -\mathbf{F}^{1}
\end{array}\right)\left(\begin{array}{l}
\beta^{0} \\
\beta^{1}
\end{array}\right)=0
$$

where $\mathbf{F}^{0}$ and $\mathbf{F}^{1}$ are $M \times M$ matrices with elements

$$
F_{m, m^{\prime}}^{0}=\Lambda_{m^{\prime}} \delta_{m, m^{\prime}}+B_{m, m^{\prime}}^{(1)}+B_{m, m^{\prime}}^{(2)}
$$

and

$$
F_{m, m^{\prime}}^{1}=\Lambda_{m^{\prime}} \delta_{m, m^{\prime}}-\Delta_{z}^{m^{\prime}} B_{m m^{\prime}}^{(1)}
$$

respectively.

$\dagger$ Equation (4) differs from his equation (17) by the appearance of a factor $m$ in the exponential. The insertion of an extra factor of $m$ in the exponential makes this equation consistent with equation (39) of his paper. 
$B_{m m^{\prime}}^{(1)}$ and $B_{m m^{\prime}}^{(2)}$ contain information about the nearest neighbour and more distant neighbour radial distributions, respectively. $\Delta_{z}$ measures the degree of angular correlation. $\Lambda_{m}$ gives the scattering strength of the single site potential. Noting that

$$
\left(\begin{array}{cc}
\mathbf{F}^{0} & \mathbf{F}^{1} \\
\mathbf{F}^{0} & -\mathbf{F}^{1}
\end{array}\right)=\left(\begin{array}{rr}
1 & 1 \\
1 & -1
\end{array}\right)\left(\begin{array}{ll}
\mathbf{F}^{0} & 0 \\
0 & \mathbf{F}^{1}
\end{array}\right)
$$

and that

$$
\operatorname{det}\left|\begin{array}{rr}
1 & 1 \\
1 & -1
\end{array}\right| \neq 0
$$

equation (6) may be written in the form

$$
\left(\begin{array}{ll}
\mathbf{F}^{0} & 0 \\
0 & \mathbf{F}^{1}
\end{array}\right)\left(\begin{array}{l}
\beta^{0} \\
\beta^{1}
\end{array}\right)=0 .
$$

This set of equations has two types of solutions. First, a solution is obtained when

$$
\begin{aligned}
\operatorname{det} \mathbf{F}^{0} & =0 \\
\beta^{0} & \neq 0 \\
\operatorname{det} \mathbf{F}^{1} & \neq 0 \\
\beta^{1} & =0 .
\end{aligned}
$$

Second, a solution is obtained when

$$
\begin{aligned}
\operatorname{det} \mathbf{F}^{0} & \neq 0 \\
\beta^{0} & =0 \\
\operatorname{det} \mathbf{F}^{1} & =0 \\
\beta^{1} & \neq 0 .
\end{aligned}
$$

(The case when both det $\mathbf{F}^{0}=0$ and $\operatorname{det} \mathbf{F}^{1}=0$ will not occur in general).

These conditions for an eigenvalue are compared with those which are obtained from a standard KKR calculation for a perfect crystal with two identical atoms per unit cell For $\boldsymbol{k}=0$ these conditions are

$$
\operatorname{det}\left|B_{m m^{\prime}}^{j j}+B_{m m^{\prime}}^{j j^{\prime}}+\Lambda_{m} \delta_{m m^{\prime}}\right|=0
$$

and

$$
\operatorname{det}\left|B_{m m^{\prime}}^{i j}-B_{m m^{\prime}}^{j j^{\prime}}+\Lambda_{m} \delta_{m m^{\prime}}\right|=0
$$

where $B_{m m^{\prime}}^{j j}$ and $B_{m m^{\prime}}^{j j^{\prime}}$ are the structure factors for the equivalent sites and nonequivalent sites, respectively. The similarity of equation (14) with equation (12a) and equation (15) with equation $(13 c)$ is noted. In particular, the presence of a similar minus sign in equations (15) and (13c) leads to the conclusion that the bonding-antibonding character of the eigenfunctions which exist in the perfect crystal at $k=0$ is also found in the disordered case. Since the bonding-antibonding character may lead to a band edge in the ordered case, one might expect analogous behaviour in the electronic energy spectrum in the disordered case. It should be noted that this analogy is made possible by the increase in the number of variational parameters due to the undetermined coefficients in the generalized rotation operator. 


\section{Criticism}

The properties ascribed by Fletcher (1967 b) to the generalized rotation operators allow the determination of the set of $a_{n, m}$. As is well known, the additivity and commutativity relation given by equation (2) plus the differentiability of the operator with respect to $\alpha$ implies that

$$
R_{m m^{\prime}}(\alpha)=\exp \left[i\left\{q(m) \alpha+\gamma_{m}\right\}\right] \delta_{m m^{\prime}}
$$

(Messiah 1962) where $\gamma_{m}$ is a phase and $q(m)$ is a constant independent of $\alpha$. The additional condition that $R(0)=1$ (equation (3)) implies that $\gamma_{m}=0$. Thus, the 'arbitrary' coefficients in equation (4) must be given by

$$
a_{n, m}=\delta_{n, n^{\prime}(m)}
$$

where $n^{\prime}$ is an integer which may be a function of $m$.

Hence, for a single generalized rotation operator (specified by giving the set of $n^{\prime}$ as a function of $m$ in equation (17)), only $M$ of the variational parameters $\beta_{n, m}$, are different from zero. The number of homogeneous equations required is $M$, not $N \times M$; and the argument which generates $N-1$ additional equations is not required. In addition, it is impossible to compare the results from a single generalized rotation operator with the result from a standard KKR calculation for a perfect crystal with two atoms per unit cell since the determinental conditions which are obtained in the two cases have different orders.

However, the solutions obtained by Fletcher are consistent with the above noted requirements on the $a_{n, m}$. For, when the solution is given by equations (12), then equation (12d) is satisfied by taking all the

$$
a_{1, m}=0
$$

and equation $(12 b)$ is consistent with taking all the

$$
a_{0, m}=1 \text {. }
$$

When the solution is given by equations (13), then equation $(13 b)$ is satisfied by taking all the

$$
a_{0, m}=0
$$

and equation (13d) is consistent with taking all the

$$
a_{1, m}=1 \text {. }
$$

The authors believe that this consistency is not due to the original assumptions about $R(\alpha)$ but is directly related to the manner in which the set of $N \times M$ homogeneous equations were obtained from $M$ homogeneous equations.

The question then arises as to whether one might view Fletcher's results as coming from two separate generalized rotation operators with the $a_{n, m}$ given by equations (18) and equations (19), respectively. Then perhaps the solution and analogy would be valid. The answer to this question seems to be no. For, when the solution and analogy are studied carefully, other difficulties appear which seem to invalidate the analogy.

Comparison of the definitions of the structure factors in equations (7) and (8) with those in equations (14) and (15) indicates that even though they are labeled in a similar manner they are somewhat different. Neither $B_{m m^{\prime}}^{j j}$ nor $B_{m m^{\prime}}^{j j^{\prime}}$ which contain information about the location of atoms throughout the whole crystal, are directly analogous to $B_{m m}^{(1)}$, which only contains information about the average nearest neighbour locations. This dependence of $B_{m m}^{(1)}$ leads to the rather unusual result that eigenvalues produced by a solution to equations (13) will depend only on the average nearest neighbour structure in contrast with the case of a perfect solid where the eigenvalues are dependent upon the location of all the atoms in the crystal. 
The weakness of the analogy is further demonstrated by looking at the variation of the wave function from site to site using transformation (1) and the solutions for the $a_{n, m}$ given in equations (18) and (19). The wave functions at $\boldsymbol{k}=0$ are found to exhibit a character which is different from the standard bonding-antibonding character found in the case of a perfect crystal. To illustrate this point, consider a wave function with only a $m=0 \mathrm{com}$ ponent. In the case where $a_{0,0}=1$ and $a_{1,0}=0$, the generalized rotation operator reduces to the unit operator. Thus,

$$
\psi_{j}(\boldsymbol{\rho})=\psi_{i}(\boldsymbol{\rho})
$$

and the wave function is of the bonding type. However, in the case where $a_{0,0}=0$ and $a_{1,0}=1$ the generalized rotation operator is just the ordinary rotation operator. Since the $m=0$ component is invariant under rotation,

$$
\psi_{j}(\rho)=\psi_{i}(\rho)
$$

and again one has a bonding solution. Thus, the two solutions are both bonding like. If the analogy were valid, one would expect one of these to be of antibonding character. The addition of higher $m$ values does not resolve this point, and results in a wave function which in the case of equations (19) may not be identifiable as either bonding or antibonding. Hence, the analogy hardly hardly seems valid. It should be noted that for a perfect crystal the antibonding s-wave functions about various sites are not related by a simple rotation.

\section{Conclusion}

The above-stated difficulties force the authors to conclude that the formalism and analogy used by Fletcher suffers from some rather fundamental inconsistencies which are of such magnitude as to question seriously the validity of this approach to the discussion of the energy spectrum of amorphous covalent semiconductors.

\section{Acknowledgment}

We would like to acknowledge Professor J. M. Ziman for suggesting this area of investigation and Professor N. H. Fletcher for his correspondence with us.

\section{References}

Fletcher, N. H., 1967 a, Adv. Phys., 16, 703-4.

1967 b, Proc. Phys. Soc., 91, 724-35. 1967 c, Proc. Phys. Soc., 92, 265-7.

KOHN, W., and ROSTOKER, N., 1954, Phys. Rev., 94, 1111-20.

Messiah, A., 1962, Quantum Mechanics (Amsterdam: North Holland) Pp 653-4.

Phariseau, P., and Ziman, J. M., 1963, Phil. Mag., 8, 1487-50. 